\title{
Evaluation of Venture Capital Based on Evaluation Model
}

\author{
Hengqing Tong, Yichao Pan, Yang Ye, Shudan Lu, Hengwen Liu \\ Department of Mathematics, Wuhan University of Technology, Wuhan, China \\ E-mail: \{tonghengqing, pych1220\}@126.com \\ Received March 28, 2010; revised April 16, 2010; accepted April 28, 2010
}

\begin{abstract}
This paper studies evaluation problem in venture capital. Based on the venture capital and the actual evaluation work, we use an evaluation model proposed by us to evaluate the profitability of enterprises. We establish the impact of investment income and investment risk index system, corresponding to get observational data of the second order indexes. Evaluation model is a kind of generalized linear regression model with convex constraint, in which the dependent variable is unknown and regression coefficients all are calculated in accordance with samples instead of the prior designated. The least squares estimation of the model is given by the interactive projection algorithm between the convex sets, so as to provide a new analysis method for venture capital evaluation index system.
\end{abstract}

Keywords: Venture Capital, Evaluation Model, Least Squares Estimation, Interactive Projection Algorithm

\section{Introduction}

The so-called venture capital assessment is to give an assessment of the benefits and the risk of investment projects according to the commercial prospectus based on the information collection [1]. Evaluation index system is the tool and method of the project evaluation, as a support system for investment appraisal. It links up the investment appraisal and assessment objective organically, and plays a role as a bridge and link, which is indispensable in investment appraisal.

Foreign countries have already set up comparatively perfect assessment index system of venture capital. In our country, venture capital is still a new thing, which has not set up comparatively perfect assessment index system yet, so for promoting development of venture capital in China, it is necessary to set up an assessment index system that is suitable for China's actual conditions in venture capital.

\section{Venture Capital Evaluation Index System}

The assessment index system of venture capital is an indispensable tool and method in the evaluation process of the venture capital. Scientific and effective assessment index system can help the professional personnel of venture capital to assess venture projects, according to the principle of investment, risk partiality and certain assessment criterion and method. It can exclude projects whose risks are too high or profits are too low among a large number of projects, and choose the investment project that has most appreciable potentiality [2].

According to the economic characteristics, we propose the impact factors of China's venture capital assessment and the main index system as shown in Table 1.

When we build the index system of venture capital assessment, we can get the data record of each index, and then the index should be gathered. In general, the gathering coefficients or weighted coefficients are assigned beforehand. In this paper we consider the weighted coefficients are calculated by samples but not man-made. Thus we should introduce a mathematical model for evaluation.

\section{Evaluation Model}

In our evaluation model there are $m$ objects to be evaluated, $p$ indexes for evaluation, and $n$ evaluates scores for each object and each index. We need to gather the evaluation score by weighted coefficients $\beta_{j}$ $(j=1, \cdots p)$ but which are unknown.

$p$ indexes are variables and noted as $x_{(1)}, \cdots x_{(p)}$. The evaluation value in $i$ th $(i=1, \cdots, n)$ evaluation for $k$ th $(k=1, \cdots, m)$ object and by $j$ th $(j=1, \cdots p)$ index is noted as $x_{i j k}$. The evaluation value data with $p$ dimension for an object in an evaluation is a row in the data matrix. There are $n$ rows for an object and is a 
data block. There are $m$ data blocks in the data matrix $X=\left\{x_{i j k}\right\} . X$ is a cubic matrix and can be arranged as different form by the regulation of cubic matrix. In this paper we arrange $X$ as $m \times n$ rows with $p$ columns. The score of the last evaluation for each object is $y_{k}(k=1, \cdots, m)$, and they are unknown also.

The evaluation model with unknown dependent variable is a generalized linear regression model. We must add some constraint for the model to solve it. Of course the weighted coefficients must satisfy $\beta_{j} \geq 0$, $j=1, \cdots, p \quad$ (i.e. $\beta \geq 0$ ), and $\beta_{1}+\beta_{2}+\cdots+\beta_{p}=1$ (i.e. $1_{p}^{\prime} \beta=1$ ). This is a prescription constraint. The dependent variables must also satisfy some constraints. Obviously we only give a unique score for each evaluation object. That is, we only give $m$ score although there $m \times n$ rows in the matrix. We define dependent variable $Y=D y$, here $D y=y \otimes 1_{n}, D_{m n \times m}=I_{m} \otimes 1_{n}$, $1_{n}=(1, \cdots, 1)^{\prime}, \quad y=\left(y_{1}, \cdots, y_{m}\right)^{\prime}$, and $\otimes$ is Kronecker product.

Thus the evaluation model may be expressed as following:

$$
\begin{gathered}
D y=X \beta+\varepsilon, E(\varepsilon)=0, \operatorname{Var}(\varepsilon)=\sigma^{2} I \\
1_{p}^{\prime} \beta=1, \quad \beta>0, D_{m n \times m}=I_{m} \otimes 1_{n}
\end{gathered}
$$

This model is proposed by us in [3]. The data structure of evaluation model is as the Table 2 .

\section{The Least Square Solution of the Generalized Linear Regression Model with Convex Constraint}

We'll discuss the LSE of the evaluation model in three steps [4-6].

Firstly, if $D y$ is known, it is just an ordinary constraint regression model. Only considering the constraint $1_{p}^{\prime} \beta=1$, we can get an explicit solution by the method of Lagrange Multiplier. Let

$$
\begin{gathered}
Q(\beta, y)=(D y-X \beta)^{\prime}(D y-X \beta) \\
\phi(\beta, y)=Q-2 \lambda\left(1_{p}^{\prime} \beta-1\right)
\end{gathered}
$$

where $\lambda$ be the multiplier. We have

$$
\begin{gathered}
\frac{\partial \phi}{\partial \beta}=-2 n X_{0}^{\prime} y+2 X^{\prime} X \beta-2 \lambda 1_{p}^{\prime}=0 \\
\frac{\partial \phi}{\partial y}=2 n y-2 n X_{0}^{\prime} \beta=0
\end{gathered}
$$

\begin{tabular}{|c|c|}
\hline \multicolumn{2}{|c|}{ Investment income index system } \\
\hline \multirow{4}{*}{$\begin{array}{c}\text { Enterprise Management } \\
\text { Level }\end{array}$} & Enterprise's strategic objectives \\
\hline & $\begin{array}{l}\text { Corporate business culture and } \\
\text { philosophy } \\
\text { The organizational structure of } \\
\text { enterprises }\end{array}$ \\
\hline & Staff wages and benefits \\
\hline & Company personnel reserve \\
\hline \multirow{5}{*}{ Entrepreneurship } & Technological innovation ability \\
\hline & Team control \\
\hline & Adaptability to the market \\
\hline & Ability to foresee the risk \\
\hline & Social interaction \\
\hline \multirow{4}{*}{ Market Environment } & Venture in which industry \\
\hline & Size of the market \\
\hline & Market growth \\
\hline & Market competition \\
\hline \multirow{3}{*}{$\begin{array}{l}\text { Products and Technical } \\
\text { Characteristics }\end{array}$} & The level of technology patents \\
\hline & Product adaptation \\
\hline & Product uniqueness \\
\hline \multirow{2}{*}{ Earning Capacity } & The level of return \\
\hline & Earnings potential growth \\
\hline \multirow{4}{*}{$\begin{array}{c}\text { Management Capacity of } \\
\text { Enterprises }\end{array}$} & management decision-making \\
\hline & $\begin{array}{l}\text { The management of enterprise } \\
\text { mobility } \\
\text { Marketing capability }\end{array}$ \\
\hline & Adaptability to the market \\
\hline & Financial risk \\
\hline \multirow{4}{*}{ Technical Risk } & Intellectual property \\
\hline & Technological advance \\
\hline & Technology alternatives \\
\hline & $\begin{array}{l}\text { The degree to prevent imitation of } \\
\text { technology } \\
\text { Technology and policy and indus- } \\
\text { try standards compliance }\end{array}$ \\
\hline \multirow{4}{*}{ Market Risk } & Market stability \\
\hline & $\begin{array}{l}\text { The difficulty of market develop- } \\
\text { ment }\end{array}$ \\
\hline & Competitor status \\
\hline & Policy trends \\
\hline \multirow{3}{*}{ Exit Risk } & Exit channel status \\
\hline & The time might withdraw \\
\hline & The way could pull out \\
\hline
\end{tabular}

where
Table 1. Venture capital evaluation index system.

$$
X_{0}^{\prime}=\frac{1}{n}\left(I_{m} \otimes 1_{n}^{\prime}\right) X=\frac{1}{n} D^{\prime} X
$$

It is a compression matrix by taking the average value of each column for each data block in matrix $X_{(m n \times p)}$. Then

$$
\hat{y}=X_{0}^{\prime} \beta
$$

Let 
Table 2. The data structure of evaluation model.

\begin{tabular}{lcccc}
\hline & $x_{(1)}$ & $x_{(2)}$ & $\ldots \ldots$ & $x_{(p)}$ \\
& $\beta_{1}$ & $\beta_{2}$ & $\ldots \ldots$ & $\beta_{p}$ \\
\hline$y_{1}$ & $x_{111}$ & $x_{121}$ & $\ldots \ldots$ & $x_{1 p 1}$ \\
$y_{1}$ & $x_{211}$ & $x_{221}$ & $\ldots \ldots$ & $x_{2 p 1}$ \\
$\vdots$ & $\vdots$ & $\vdots$ & $\vdots$ & $\vdots$ \\
$y_{1}$ & $x_{n 11}$ & $x_{n 21}$ & $\ldots \ldots$ & $x_{n p 1}$ \\
\hline$\vdots$ & $\ldots \ldots$ & $\ldots \ldots$ & $\ldots \ldots$ & $\ldots \ldots$ \\
$\vdots$ & $\ldots \ldots$ & $\ldots \ldots$ & $\ldots \ldots$ & $\ldots \ldots$ \\
$\vdots$ & $\ldots \ldots$ & $\ldots \ldots$ & $\ldots \ldots$ & $\ldots \ldots$ \\
\hline$y_{m}$ & $x_{11 m}$ & $x_{12 m}$ & $\ldots \ldots$ & $x_{1 p m}$ \\
$y_{m}$ & $x_{21 m}$ & $x_{22 m}$ & $\ldots \ldots$ & $x_{2 p m}$ \\
$\vdots$ & $\vdots$ & $\vdots$ & $\vdots$ & $\vdots$ \\
$y_{m}$ & $x_{n 1 m}$ & $x_{n 2 m}$ & $\ldots \ldots$ & $x_{n p m}$ \\
\hline
\end{tabular}

$$
P_{D}=I_{m n}-\frac{1}{n} D D^{\prime}
$$

It is easy to verify that $P_{D}$ is a projection matrix. Since $\left(X^{\prime} X-n X_{0}^{\prime} X_{0}\right) \beta=\lambda 1_{p}$, letting

$$
A=X^{\prime} X-n X_{0}^{\prime} X_{0}^{\prime}=X^{\prime} P_{D} X
$$

when $A$ is invertible, the solution of $\beta$ is

$$
\hat{\beta}=\lambda A^{-1} \mathbf{1}_{p}^{\prime}=\frac{A^{-1} \boldsymbol{1}_{p}}{\mathbf{1}_{p}^{\prime} A^{-1} \mathbf{1}_{p}^{\prime}}
$$

Summarizing the aforesaid, we have the following theorem.

THEOREM 1. If $D y$ is known and $r k\left(P_{D} X\right)=p$, under the constrain $1_{p}^{\prime} \beta=1$,

$$
Q(\beta, y)=\|D y-X \beta\|^{2} \stackrel{y, \beta}{\longrightarrow} \min
$$

has unique solution (8) and (11). If each component of $\hat{\beta}$ is nonnegative, (8) and (11) are also the solution of the evaluation model (1) (2).

Secondly, if some components of $\hat{\beta}$ are negative, we must consider the constraints $1_{n}^{\prime} \beta=1$ and $\beta>0$ simultaneously. Then it is a prescription regression model. For the existence and uniqueness of the solution of the model (1) (2), we have the following theorem.

THEOREM 2. If $r k(D \mid X)=m+p$, the evaluation model has unique solution.

The proof is easy when we rewrite (12) as

$$
\left\|0-(D \vdots X)\left(\begin{array}{c}
-y \\
\beta
\end{array}\right)\right\|^{2} \stackrel{y, \beta}{\longrightarrow} \min
$$

the set

$$
\begin{array}{r}
A_{D X}=\left\{(D \vdots X)\left(\begin{array}{c}
-y \\
\beta
\end{array}\right) \mid y \in R^{m},\right. \\
\left.1_{p}^{\prime} \beta=1, \beta \geq 0, \beta \in R^{p}\right\}
\end{array}
$$

is a closed set, so there exists an unique point $A_{0} \in A_{D X}$ and $A_{0}$ satisfies (13). Because the column of $(D \mid X)$ is full rank, we can obtain unique solution of $y$ and $\beta$ from $A_{0}=X \beta-D y$.

Thirdly, we'll discuss the algorithm of the model when $D y$ is unknown. We consider the geometric background of (12). Denote sets

$$
\begin{gathered}
A=\left\{D y \mid y \in R^{m}\right\} \\
B=\left\{X \beta \mid 1_{p}^{\prime} \beta=1, \beta \geq 0, \beta \in R^{p}\right\}
\end{gathered}
$$

The Formula (12) means to seek the shortest Euclidean distance between sets $A$ and $B$. Obviously, $A$ and $B$ are two closed convex sets and $B$ is bounded. According to the theorem "the distance between two closed convex sets can be reached", the solution of (12) exists.

How to find the shortest distance between two convex sets? We consider the method of the alternating projection between two sets. Let $A_{0}$ be a point in the set $A$. If $d\left(A_{0}, B_{0}\right)=d\left(A_{0}, B\right)$, we call $B_{0}$ the projection from the point $A_{0}$ to the set $B$. The following is the alternating projection process.

Take an arbitrary initial value $A_{0} \in A$, find $B_{0} \in B$, satisfying $d\left(A_{0}, B_{0}\right)=d\left(A_{0}, B\right)$. For $B_{0}$, take $A_{1} \in A$, satisfying $d\left(B_{0}, A_{1}\right)=d\left(B_{0}, A\right)$. For $A_{i} \in A$, take $B_{1} \in B$, satisfying $d\left(A_{i}, B_{i}\right)=d\left(A_{i}, B\right)$. For $B_{i}$, take $A_{i+1} \in A$, satisfying $d\left(B_{i}, A_{i+1}\right)=d\left(B_{i}, A\right)$, and so on. When $d\left(A_{i}, B_{i}\right)<\varepsilon$, iterative process is stopped and computation is completed. The meaning of convergence of aforesaid iterative process is:

$$
\lim _{i \rightarrow \infty} d\left(A_{i}, B_{i}\right)=d(A, B)=d\left(A^{*}, B^{*}\right)
$$

where $A^{*}, B^{*}$ are two points which belong to sets $A$ and $B$ respectively.

In one word, the distance between two closed convex sets may be obtained by making use of successive computation of distance between a point and a closed convex set. In the evaluation model consisting of (1) (2), for arbitrary $y_{i}$, we can get the solution of $\beta_{i}$ according to the Theorem 1 and 2. For the solution of $\beta_{i}$, (1) becomes a common multivariate regression model which can be solved. The convergence of the alternating projection iterative process has been proved in [3] and is omitted here. 


\section{Example}

The following example is given by the DASC software developed by ourselves. The evaluation model data has 30 rows and 6 columns. Evaluation marks are all set from 1 to 10. The martix of evalution data is as the Table 3.

The computation process shows the convergence process is very fast and it has iterated 7 times altogether. The subprogram gives an initial value $y_{1}=y_{2}=y_{3}=10$ and a control precision 0.0001 . The sum of regression coefficients should be 1 in the printing result of iterative process.

Notice that the three different fitted values of $y$ are the evaluation results actually. And the observation value of $y$ is calculated according to fitting equation. The fitting effect figure provides a visual awareness of

Table 3. The martix of evalution data.

\begin{tabular}{ccccccc}
\hline & $\beta_{1}$ & $\beta_{2}$ & $\beta_{3}$ & $\beta_{4}$ & $\beta_{5}$ & $\beta_{6}$ \\
\hline$Y_{1}$ & 1 & 2 & 8 & 5 & 6 & 3 \\
$Y_{1}$ & 1 & 7 & 7 & 10 & 4 & 6 \\
$Y_{1}$ & 9 & 1 & 1 & 6 & 7 & 1 \\
$Y_{1}$ & 4 & 1 & 5 & 7 & 6 & 10 \\
$Y_{1}$ & 9 & 6 & 1 & 7 & 5 & 8 \\
$Y_{1}$ & 10 & 8 & 3 & 1 & 8 & 4 \\
$Y_{1}$ & 7 & 8 & 10 & 4 & 3 & 10 \\
$Y_{1}$ & 8 & 8 & 7 & 1 & 7 & 9 \\
$Y_{1}$ & 3 & 5 & 8 & 5 & 3 & 3 \\
$Y_{1}$ & 4 & 2 & 5 & 9 & 10 & 1 \\
\hline$Y_{2}$ & 10 & 6 & 6 & 4 & 10 & 5 \\
$Y_{2}$ & 3 & 1 & 10 & 1 & 6 & 4 \\
$Y_{2}$ & 3 & 10 & 6 & 5 & 10 & 1 \\
$Y_{2}$ & 8 & 8 & 9 & 2 & 1 & 7 \\
$Y_{2}$ & 9 & 7 & 8 & 8 & 10 & 9 \\
$Y_{2}$ & 3 & 4 & 4 & 6 & 6 & 9 \\
$Y_{2}$ & 5 & 9 & 3 & 5 & 6 & 5 \\
$Y_{2}$ & 3 & 2 & 2 & 6 & 9 & 1 \\
$Y_{2}$ & 6 & 5 & 10 & 8 & 6 & 9 \\
$Y_{2}$ & 7 & 9 & 2 & 3 & 8 & 2 \\
\hline$Y_{3}$ & 1 & 3 & 1 & 5 & 1 & 8 \\
$Y_{3}$ & 10 & 3 & 2 & 4 & 9 & 7 \\
$Y_{3}$ & 2 & 7 & 4 & 4 & 5 & 2 \\
$Y_{3}$ & 6 & 9 & 6 & 10 & 6 & 2 \\
$Y_{3}$ & 10 & 5 & 2 & 6 & 3 & 5 \\
$Y_{3}$ & 5 & 10 & 2 & 2 & 4 & 7 \\
$Y_{3}$ & 2 & 7 & 7 & 9 & 3 & 5 \\
$Y_{3}$ & 4 & 3 & 1 & 10 & 5 & 2 \\
$Y_{3}$ & 10 & 5 & 2 & 9 & 1 & 2 \\
$Y_{3}$ & 1 & 4 & 3 & 2 & 8 & 5 \\
\hline & & & & & & \\
\hline
\end{tabular}

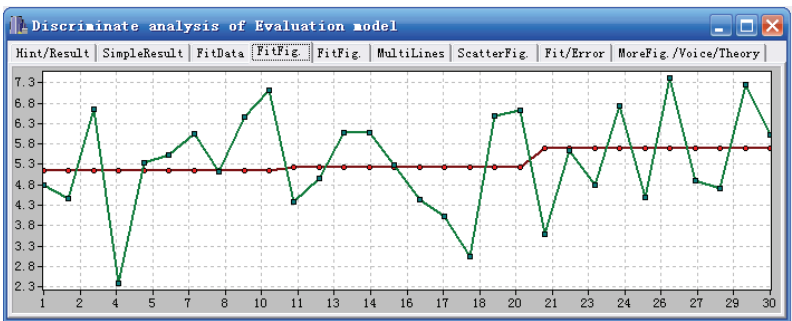

Figure 1. The fitting effect figure.

evaluation model. Results are $y=5.1451,5.2407$, 5.6958), $\beta=(0.2298,0.2106,0.1510,0.0588,0.2457$, 0.1041 ), where $y$ is the final evaluation mark and $\beta$ is the regression coefficient, also called weight coefficient.

Evaluation model can be used as the basis for clustering. When we input $X=(2,3,4,5,6,7)$ in the prediction program, the forecast value is 5.1190 . It denotes that this group data should attribute to the fist group, because the difference between 5.1190 and 5.1451 is the minimum.

\section{Conclusions}

In this paper, we studied the index system of risk evaluation for venture capital, and gave the algorithms of model in which the gathering coefficients of index were calculated by samples. Our algorithm also provides a solution scheme for some problems in venture capital evaluation, so this paper gives a new idea for venture capital evaluation with some practical reference value.

\section{References}

[1] Z. W. Zhao, "Research on the Appraisal and DecisionMaking in Venture Capital," Management Science and Engineering (Professional), Tianjin University, Doctoral Dissertation, 2005.

[2] Z. H. Wang and L. Li, "Comparison of Venture Capital Assessment," Journal of Nanhua University (Social Science Edition), Vol. 8, No. 1, February 2007, pp. 43-45.

[3] H. Q. Tong, "Evaluation Model and its Iterative Algorithm by Alternating Projection," Mathematical and Computer Modelling, Vol. 18, No. 8, 1993, pp. 55-60.

[4] H. Q. Tong, S. J. Zhong, T. Z. Liu and Y. F. Deng, "Biostatistics Algorithm: Evaluation Model with Convex Constraint and its Parameters Estimates," The 1st International Conference on Bioinformatics and Biomedical Engineering, 2007, pp. 402-405.

[5] H. Q. Tong, "Theory of Economics," Science Press, 2005, pp. 262-264.

[6] K. T. Fang and S. D. He, "Regression Models with Linear Constraints and Nonnegative Regression Coefficients," Mathematica Numerica Sinica, Vol. 7, 1985, pp. 97-102. 